\title{
Effectiveness of Ethanol infusion into the vein of Marshall combined with a fixed anatomical ablation strategy (the 'upgraded 2C3L' approach) for catheter ablation of persistent atrial fibrillation
}

Yi-wei $\mathrm{Lai}^{1}$, Xiaoxia $\mathrm{Liu}^{2}$, Caihua Sang ${ }^{3}$, Deyong Long ${ }^{3}$, Mengmeng $\mathrm{Li}^{1}$, Weili $\mathrm{Ge}^{4}$, Xiangfei $\mathrm{Liu}^{5}$, Zhibing $\mathrm{Lu}^{6}$, Qi Guo ${ }^{1}$, Chao Jiang ${ }^{7}$, Song Zuo ${ }^{1}$, Chen-Xi Jiang ${ }^{8}$, Rong Bai ${ }^{7}$, Ribo Tang ${ }^{3}$, Xue-Yuan Guo ${ }^{8}$, Li Song-Nan ${ }^{9}$, Nian Liu ${ }^{10}$, Wei Wang ${ }^{7}$, Xin Zhao ${ }^{7}$, Changyi $\mathrm{Li}^{1}$, Xin $\mathrm{Du}^{8}$, Jian zeng Dong ${ }^{11}$, and Chang Sheng $\mathrm{Ma}^{7}$

${ }^{1}$ Capital Medical University Affiliated Anzhen Hospital

${ }^{2}$ Harbin Medical University Fourth Hospital

${ }^{3}$ Capital Medical University

${ }^{4}$ Taizhou Hospital of Zhejiang Province

${ }^{5}$ Shengli oilfield Central Hospital

${ }^{6}$ Wuhan university

${ }^{7}$ Beijing An Zhen Hospital

${ }^{8}$ Beijing An Zhen Hospital, Capital Medical University

${ }^{9}$ Beijing Anzhen Hospital,Capital Medical University

${ }^{10}$ Capital University of Medical Sciences

${ }^{11}$ Beijing Anzhen Hospital Capital Medical University

March 27, 2021

\begin{abstract}
Abstract Introduction: Linear ablation in addition to pulmonary vein antrum isolation (PVAI) has failed to improve the success rate for persistent atrial fibrillation (PeAF), due to incomplete block of ablation lines, especially in the mitral isthmus (MI). Methods and results: The study enrolled 191 patients (66 in group 1 and 125 in group 2). In group 1, EI-VOM was firstly performed, followed by radiofrequency (RF) applications targeting bilateral PVAI and bidirectional block in the roofline, cavotricuspid isthmus, and MI. In group 2, PVAI and the three linear ablations were completed using only RF energy. MI block was achieved in 63(95.5\%) and 101(80.8\%) patients in group 1 and 2 , respectively $(\mathrm{p}=0.006)$. Patients in group 1 had shorter ablation time for left pulmonary vein antrum $(8.15 \mathrm{~min}$ vs $12.59 \mathrm{~min}, \mathrm{p}<0.001)$ and $\mathrm{MI}(7.0 \mathrm{~min}$ vs $11.8 \mathrm{~min}, \mathrm{p}<0.001)$ and required less cardioversion $(50(78.5 \%)$ vs $113(90.4 \%), \mathrm{p}=0.007)$. During the 12 -month follow-up, $58(87.9 \%)$ patients were free from $\mathrm{AF} / \mathrm{AT}$ in group 1 compared with $81(64.8 \%)$ in group $2(\mathrm{p}<0.001)$. In multivariate cox regression, the 'upgraded $2 \mathrm{C} 3 \mathrm{~L}$ ' procedure is associated with a lower recurrence rate (HR $0.27,95 \%$ CI $0.12-0.59$ ). Conclusion: Compared with the conventional '2C3L' approach, the 'upgraded 2C3L' approach has higher effectiveness for ablation of PeAF.
\end{abstract}

Effectiveness of Ethanol infusion into the vein of Marshall combined with a fixed anatomical ablation strategy (the 'upgraded 2C3L' approach) for catheter ablation of persistent atrial fibrillation

Yiwei Lai*1, MD, Xiaoxia Liu*1, MD, Caihua Sang\# ${ }^{1}$, MD, Deyong Long ${ }^{1}$, MD, Mengmeng Li $^{1}$, MD, Weili $\mathrm{Ge}^{2}, \mathrm{MD}$, Xiangfei Liu ${ }^{3}$, MD, Zhibing $\mathrm{Lu}^{4}$, MD, Qi Guo, MD, Chao Jiang ${ }^{1}$, MD, Song Zuo ${ }^{1}$, MD, Chenxi 
Jiang ${ }^{1}$, MD, Rong Bai ${ }^{1}$, MD, Ribo Tang ${ }^{1}$, MD, Xueyuan Guo ${ }^{1}$, MD, Songnan $\mathrm{Li}^{1}$, MD, Nian Liu ${ }^{1}$, MD, Wei Wang $^{1}$, MD, Xin Zhao ${ }^{1}$, MD, Changyi $\mathrm{Li}^{1}$, MD, Xin $\mathrm{Du}^{1}$, MD, Jianzeng Dong ${ }^{1}$, MD, Changsheng Ma\# ${ }^{1}$, MD

\title{
Affiliations:
}

1. Department of Cardiology, Beijing Anzhen Hospital, Capital Medical University; National Clinical Research Center for Cardiovascular Diseases

2. Department of Cardiology, Taizhou Hospital of Zhejiang Province, Taizhou, China.

3. Department of Cardiology, Shengli oilfield Central Hospital, Shandong, China.

4. Department of Cardiology, Zhongnan Hospital of Wuhan University, Wuhan, China.

*YW-L and XX-L contribute equally to this work

\#Co-correspondance to:

1.Caihua Sang, MD, Department of Cardiology, Beijing Anzhen Hospital, Capital Medical University, No. 2, Anzhen Road, Chaoyang District, 100029 Beijing, China.

E-mail: sch9613070@sina.com

2. Changsheng Ma, MD, Department of Cardiology, Beijing Anzhen Hospital, Capital Medical University, No. 2, Anzhen Road, Chaoyang District, 100029 Beijing, China. E-mail:chshma@vip.sina.com

\begin{abstract}
Introduction: Linear ablation in addition to pulmonary vein antrum isolation (PVAI) has failed to improve success rate for persistent atrial fibrillation (PeAF), due to incomplete block of ablation lines, especially in the mitral isthmus (MI).

Methods and results: The study enrolled 191 patients (66 in group 1 and 125 in group 2). In group 1, EI-VOM was firstly performed, followed by radiofrequency (RF) applications targeting bilateral PVAI and bidirectional block in the roofline, cavotricuspid isthmus, and MI. In group 2, PVAI and the three linear ablations were completed using only RF energy. MI block was achieved in 63(95.5\%) and 101(80.8\%) patients in group 1 and 2 , respectively $(\mathrm{p}=0.006)$. Patients in group 1 had shorter ablation time for left pulmonary vein antrum (8.15 min vs $12.59 \mathrm{~min}, \mathrm{p}<0.001)$ and $\mathrm{MI}(7.0 \mathrm{~min}$ vs $11.8 \mathrm{~min}, \mathrm{p}<0.001)$ and required less cardioversion $(50(78.5 \%)$ vs $113(90.4 \%), \mathrm{p}=0.007)$. During the 12-month follow-up, $58(87.9 \%)$ patients were free from AF/AT in group 1 compared with $81(64.8 \%)$ in group $2(\mathrm{p}<0.001)$. In multivariate cox regression, the 'upgraded 2C3L' procedure is associated with a lower recurrence rate (HR 0.27, 95\% CI 0.12-0.59).
\end{abstract}

Conclusion: Compared with the conventional '2C3L' approach, the 'upgraded 2C3L' approach has higher effectiveness for ablation of PeAF.

Keywords: atrial fibrillation, vein of Marshall, ethanol infusion, catheter ablation

\section{Introduction}

Catheter ablation has now been recommended as the first-line therapy for drug-refractory symptomatic atrial fibrillation (AF), even in persistent $\mathrm{AF}$ with major risk factors for recurrence[1]. The current cornerstone of the procedure is to completely isolate pulmonary veins. However, pulmonary vein antrum isolation (PVAI) alone is far from enough to maintain sinus rhythm in patients with persistent AF, advocating the application of atrial substrate modification.

Left atrial (LA) linear ablation is one of the most common procedures used in addition to PVAI. In our previous study, compartmentalizing the atria with the fixed ' $2 \mathrm{C} 3 \mathrm{~L}$ ' approach including bilateral PVAI and three linear ablation lesion sets across the mitral isthmus (MI), left atrial roof, and cavotricuspid isthmus (CTI) is 
comparable to stepwise ablation[2]. However, failure in MI block and high MI conduction recovery rate are the most important limitations of this approach that attribute to the recurrence of atrial tachycardias(AT). For the similar reason, the STAR AF II trial failed to prove the added value of linear ablation[3].

Ethanol infusion into the vein of Marshall (EI-VOM) has been found effective to achieve MI bidirectional block and facilitate the ablation of PMAT[4]. More recently, the VENUS trial has reported the benefit of EI-VOM in the treatment of persistent AF in addition to radiofrequency (RF) ablation[5]. However, in its post-hoc analysis, this benefit seems to be restricted to those with MI block[6]. In the present prospective study, we evaluated the effectiveness of an 'upgraded 2C3L' approach aiming to achieve a higher MI block rate, more thorough atrial compartmentation, and less AF/AT recurrence.

\section{Method}

\section{Population and study design}

The present study is approved by the ethics committee of Beijing Anzhen Hospital, and all patients provided written informed consent before the procedure. From July 2018 to Jan 2020, 191 consecutive patients with PeAF undergoing de novo ablation were enrolled. The inclusion criteria were: 1) Age over 18 years; 2) AF persisting over 3 months and less than 3 years; 3) Refractory to at least one class I or III antiarrhythmic drug; 4) No previous history of ablation for AF. Patients enrolled during Jul 2019 and Jan 2020 underwent EI-VOM combined with RF ablation using the '2C3L' approach (group 1), while 125 patients enrolled during July 2018 and June 2019 underwent only RF ablation using the '2C3L' approach (group 2). The flowchart of the study is shown in Figure 1.

\section{Intra-procedural setting}

Electrophysiological study and ablation procedure was performed under conscious sedation with fentanyl and midazolam. A deflectable decapolar catheter was positioned in the coronary sinus (CS). An 8.5-Frenchlong sheath (SL1; St. Jude Medical, Minnetonka, USA) was introduced into the LA using a modified Brockenbrough technique. To prevent thrombus formation, the transseptal sheath was continuously flushed with heparinized saline $(20 \mathrm{~mL} / \mathrm{h})$ and i.v. heparin was administered to maintain an activated clotting time of 300-350 seconds. The LA was reconstructed under the guidance of a three-dimensional electroanatomic mapping system (CARTO, Biosense-Webster, Inc.) using a high-density mapping catheter (PENTARAYß) Catheter Biosense-Webster Inc.). The catheter ablation was performed with a $3.5-\mathrm{mm}$ cool saline-irrigated ablation catheter (Thermo-Cool SMARTTOUCH SF® Catheter. Biosense-Webster Inc, Diamond Bar, CA, USA). Point-by-point application was performed using power-control mode, with a temperature limited under $43^{\circ} \mathrm{C}$ and a saline irrigation rate of $8-30 \mathrm{~mL} / \mathrm{min}$. RF energy was delivered with a power output of 25 $\mathrm{W}$ in the CS and 35-50 W elsewhere. Radiofrequency applications were depicted using automated tagging technology (Carto VisiTagTM, Biosense Webster Inc.), with a filter threshold of catheter motion $<2.5 \mathrm{~mm}$ within 4 seconds and contact force [?] $8 \mathrm{~g}$ for $70 \%$ of the time. Targets for ablation index (AI) were: (1) 500-550 for anterior wall; (2) 350-400 for posterior wall; (3) 450-500 for the LA roof and CTI; (4) 550-600 for MI.

\section{Study procedure}

Details of the procedure in each group are shown in Figure 2 and $\mathbf{3}$. EI-VOM was taken as the first step of the "upgraded 2C3L" approach in group 1. An 8.5-French-long sheath (SL1; St.Jude Medical, Minnetonka, MN, USA) is inserted into the right atrium just below the CS ostium, and a steerable long sheath (Agilis NxT; Abbott) is used if necessary. Under the right anterior oblique view, a $6 \mathrm{~F}$ guiding catheter (Judkins R4.0, Medtronic, MN) is inserted into the CS pointing posteriorly and superiorly near the Vieussens Valve in search of the VOM ostium, and then a selective VOM venogram was acquired. A BMW guiding wire (0.014 inch x $190 \mathrm{~cm}$; Abbott, MN) supported by an over-the-wire balloon (1.5-2.5 mm diameter and 8-12 mm length, Boston Scientific) catheter is advanced into the VOM. An appropriately sized balloon was gradually inflated at a maximum of 6 to $8 \mathrm{~atm}$ in the VOM. A selective venogram of the VOM is obtained by injecting $1 \mathrm{~mL}$ of contrast medium to confirm complete balloon occlusion and VOM distribution. 2-4 $\mathrm{mL}$ of ethanol 
(95\% ethanol) is slowly injected into the distal of VOM and selective venography of the VOM is repeated. After 5 minutes, in the same way, another injection is performed for the proximal and/or middle part of the VOM. The maximum ethanol volume allowed was $12 \mathrm{ml}$. The endpoint of EI-VOM is the contrast media staining colocalizing the course of VOM.

RF ablation using the '2C3L' approach is performed in both group 1 and group 2, the details of which have been described previously [2]. In brief, single-catheter technique was adopted for the ablation procedure in LA. Bilateral circumferential pulmonary vein antrum (PVA) ablation was firstly performed, followed by the roofline joining two circumferential lesions, and then posterior MI ablation was performed extending from the annulus to the left inferior pulmonary vein ostium. The CS will also be ablated if necessary. CTI ablation starts from the 6'o clock of the tricuspid valve annulus to the inferior cava vein. If an organized AT occurred during the procedure, mapping guided ablation will be performed. Cardioversion was performed if AF persists. After the restoration of SR, LAA was mapped to observe whether significant LAA activation delay was present, which was defined as the local activation of the LAA delayed beyond the onset of QRS wave.

In both groups, the endpoint of the procedure is complete PVAI and bidirectional block along all ablation lines, which was confirmed under sinus rhythm[7, 8].

\section{Follow up}

All patients were discharged with an antiarrhythmic medication and an oral anticoagulant (OAC). Antiarrhythmic drugs were stopped 3 months after ablation if the patient had no recurrence of atrial tachyarrhythmia, while OAC is prescribed according to the patient's thromboembolic risk.

Follow-up was conducted at 3, 6, and 12 months after the procedure through telephone or in the outpatient clinic. A 24-hour Holter recording was performed at 1, 2, 3, 6, 12 months. Symptom triggered or opportunistic ECGs were also documented. Recurrence was defined by an episode of atrial tachyarrhythmia lasting more than 30 seconds after a 3-month blanking period. The primary outcome of the observation is freedom from $\mathrm{AF} / \mathrm{AT}$ recurrence with or without anti-arrhythmic drugs (AAD) after the 3-month blanking period during the 12-month follow-up after single ablation.

\section{Statistical analysis}

All continuous variables are presented as mean +- SD or median (range) if not normally distributed. Continuous data were compared using Student's t-test or Mann-Whitney U-test. Categorical variables were compared by Chi-square or Fisher's exact test as appropriate. Additional Cox regression was also conducted to assess the freedom from AF/AT during follow-up. Variables of clinical importance or those with a significant intergroup difference at baseline will be included in the regression model. A P-value of 0.05 was considered statistically significant. Statistical analyses were performed using R 4.0.2.

\section{Results}

\section{Baseline characteristics}

The final population comprised of 191 patients with a mean age of 61.0+-10.5 years, 131(68.6) of them were male. Patients undergoing 'upgraded 2C3L' procedure had a higher prevalence of hypertension $(48.5 \%$ vs $22.4 \%, \mathrm{p}<0.001)$. The intergroup difference was not statistically significant in terms of age, sex, type of AF, ultrasound features, and other comorbidities. (Table 1)

\section{Procedural information}

Procedural findings are shown in Table 2 . Successful EI-VOM was performed in 53(80.3) patients in group 1 , with an average ethanol use of $6.9+-1.9 \mathrm{ml}$. Twelve patients cannot find the VOM and one had VOM dissection during selective venography that precluded ethanol infusion. The total procedure time was similar between group 1 and group $2(162.4+-39.7$ vs $171.5+-44.8, \mathrm{p}=0.170)$. Although fluoroscopy time was longer in group1 $(11.82+-8.3$ vs $4.3+-3.7, \mathrm{p}<0.001)$, ablation time was shorter in both $\mathrm{MI}(7.0+-3.5$ vs $11.8+-3.5$, 
$\mathrm{p}=0.029)$ and left PVA $(8.2+-4.00$ vs $12.6+-2.87, \mathrm{p}<0.001)$ was much shorter in group 1 . PVAI, complete roofline block, and CTI block could be achieved in all patients, except one in group 2 failed to achieve CTI block. MI bidirectional block rate was significantly higher in group $1(95.5 \%$ vs $80.8 \%, \mathrm{p}=0.006)$. Moreover, less electric cardioversion was required to restore sinus rhythm in patients undergoing EI-VOM (78.5\% vs $90.4 \%, \mathrm{p}=0.007)$. LAA conduction delay was not observed in both groups during the procedure.

In group 1, 2(3.0\%) patients had mild-to-moderate complications, including one mild pericardial effusion with self-relief and one fluid overload during the procedure. Eight complications occurred in $7(5.6 \%)$ patients in group 2, including four fluid overload, one mild pericardial effusion with self-relief, two arteria-venous fistula, and one pleural effusion. No severe complications like death, stroke, or atrial-esophagus fistula was observed in the study population.

\section{Follow up}

At the 12-month follow-up, fifty-eight (87.9\%) patients were free from AF/AT in group 1 compared with 81 $(64.8 \%)$ in group $2(\mathrm{p}<0.001)$, among them 2 in group 1 and 3 in group 2 were on AAD during the 12 -month follow-up. And for 53 patients with successful EI-VOM, freedom from AF/AT recurrence was achieved in $47(88.7 \%)$. In cox regression, patients in group 1 had higher survival freedom from AT recurrence after adjusted for age, LA diameter, LSPAF, hypertension, and heart failure (HR 0.27 95\%CI 0.12-0.59) (Figure 4 ).

Four of 8 patients with recurrent AF/AT in group 1 underwent a redo procedure. One of them had recurrent $\mathrm{AF}$, two were proved to be perimitral AFL eliminated by RF applications at the annulus side of MI and another one had a conduction gap at the RPV. In group 2, 12 of 44 patients with recurrent AF/AT underwent a redo procedure, including $4 \mathrm{AF}, 3$ perimitral AFL, 2 roofline-related flutter, 2 flutters related to RSPV conduction recovery, one scar-related reentry.

\section{Discussion}

The present study evaluated the effectiveness of a fixed, reproducible 'upgraded 2C3L' ablation strategy that combines the EI-VOM and RF ablation targeting bilateral PVAI and bidirectional linear block of MI, CTI, and LA roofline. This strategy is associated with higher sinus rhythm maintenance compared with the conventional '2C3L' approach during the 12-month follow-up.

Current guidelines have indicated that PVI alone is insufficient for maintaining sinus rhythm in patients with PeAF[1], and varieties of substrate modification strategies, including anatomical ablation (linear ablation) and electrogram guided ablation(ablation of rotors, fractionated electrograms, et al), have been widely adopted by electrophysiologists in recent decades. In surgical ablation, linear lesions have been proven to be effective in rhythm control[9]. Theoretically, linear ablation helps to achieve LA compartmentation, stop the rotors roaming around the left atrium, and therefore preventing the maintenance of $\mathrm{AF}[10]$. But the conclusion is quite different for catheter ablation. The main reason for this inconsistency lies in the fact that transmural lesions cannot always be achieved by radiofrequency catheter ablation. As is reported in the STAR AF II trial[3], where LA linear ablation involving the roofline and mitral isthmus failed to bring about higher SR maintenance compared with PVI alone, the overall linear block rate was only $74 \%$.

With the advancement of ablation technology, complete block of the roofline and CTI can now be achieved in most cases. But the ablation of the MI line remains challenging. In the ALINE study, even by using an AI-guided, point-by-point optimized RF ablation, MI bidirectional block can only be achieved in $80 \%$ of patients, while in most cases roofline can be easily blocked.[11] Meanwhile, conduction recovery across the MI line can occur in 38 of 52 patients with recurrent AF/AT[12]. Besides the atrial wall thickness and heat-sinking effect of epicardial vessels, one important cause is epicardial conduction through the ligament of Marshall[13]. In our previous study of the '2C3L' approach, perimitral atrial flutter accounts for 10 of 24 recurrent AF/ATs after ablation for PeAF [2]. Moreover, it is reported that the Marshall bundle (MB) participates in $30.2 \%$ of reentrant ATs after AF ablation. Only $81.6 \%$ of these MB-related ATs could be terminated using RF ablation, and the recurrence rate was as high as $41.3 \%$ during 18-months follow up[14]. 
EI-VOM may provide a solution to achieve MI bidirectional block. Through the VOM and its collateral flow, the ethanol can infiltrate into the atrial myocardium and rapidly cause transmural lesions that mainly involve the posterolateral LA free wall and anterior part of the left pulmonary antrum[15]. More importantly, lesions resulting from ethanol infusion has been proved to have higher durability, which prevents future reconnection across the blocked MI line[16]. For persistent atrial fibrillation, data revealing the value of EI-VOM on substrate modification is still limited. In the recently published VENUS trial, EI-VOM before $\mathrm{RF}$ ablation significantly improved long-term AF free survival and reduced AF burden compared with RF ablation alone[5]. However, as is reported in its post-hoc analysis, patients could benefit from EI-VOM only if MI was blocked[6]. Meanwhile, a prospective, single-arm study has demonstrated that EI-VOM in conjunction with a fixed strategy of LA linear ablation has ideal short- and long-term effectiveness [17]. In view of current studies, an anatomical ablation strategy targeting atrial compartmentation seems to better take advantage of EI-VOM, compared with empirical substrate modification.

Herein, we conducted a comparative study to further elucidate the value of a fixed ablation strategy that combines EI-VOM and the well-established '2C3L' approach. At the very beginning of the procedure, both the proximal and distal parts of the VOM were ablated with ethanol infusion. With EI-VOM, anatomical structures refractory to RF energy, like the Marshall bundle, left lateral ridge, and LA myocardium near epicardial vessels can be easily injured transmurally. Theoretically, it is promising in reducing the recurrence of perimitral ATs. Meanwhile, facilitated LPV antrum ablation and better atrial compartmentation by linear lesions further prevent the recurrence of atrial fibrillation. Patients with AF triggers originating from the LOM can also benefit from EI-VOM[18]. Moreover, as is observed in the present study, in this fixed, less progressive 'upgraded 2C3L' approach, ablation is mostly performed at sites with the latest atrial activation and is less likely to result in left atrial appendage conduction delay, preventing the physiology of atria. As for safety concerns, the incidence of the adverse event during EI-VOM is reasonably low in the present study, as is also reported previously[19].

It is also important to realize the limitation of EI-VOM itself. The VOM is not always accessible, and the accessible rate varies from $71.4 \%-96[5,16,17,20]$. The difference in techniques, limited sample size of the reports could be the potential explanations that require further research. Of special note, the annulus side of MI is not covered by the EI-VOM lesion. In the present study, ablation in the coronary sinus is equally required in both groups, and MI conduction gap near the annular side is the cause of recurrent perimitral flutters in group 1.

\section{Limitations}

Despite the encouraging result in the present study, it is limited by its non-randomized design and small sample size. And AF burden is not analyzed. We will continue to look for clinical evidence of higher quality in our ongoing randomized trial, the PROMPT-AF trial, which directly compares the efficacy of the "upgraded 2C3L' procedure with PVI (NCT04497376). Meanwhile, atrial physiology was only assessed by activation pattern after ablation while pre- and post-procedural ultrasound cardiography was not routinely performed. Moreover, only 4 patients in the present study underwent a redo procedure after EI-VOM, the features of recurrent tachycardias after EI-VOM remain to be further investigated

\section{Data availability}

Data is available upon reasonable request from the corresponding author.

\section{Acknowledgment}

We sincerely thank our clinical specialists, Mr. Lang Qin, Mr. Longjian Geng, and Ms. Yan Zhu from Biosense Webster, JJMC, who has been very helpful during the ablation procedure and in the collection of the CARTO backup data.

\section{Funding Sources}

This work was supported by the National Key Research and Development Program of China (grant num- 
ber:2016YFC0900901, 2016YFC1301002, 2018YFC1312501, 2017YFC0908803, and 2020YFC2004803) and the Zhongnanshan Medical Foundation of Guangdong Province.

\section{Disclosures}

Changsheng Ma has received honoraria from Bristol-Myers Squibb (BMS), Pfizer, Johnson \& Johnson, Boehringer-Ingelheim (BI), and Bayer for giving lectures. Jianzeng Dong has received honoraria from Johnson \& Johnson for giving lectures. The other authors have no conflict of interest.

\section{References:}

1. Hindricks G, Potpara T, Dagres N, Arbelo E, Bax JJ, Blomstrom-Lundqvist C, Boriani G, Castella M, Dan GA, Dilaveris PE, Fauchier L, Filippatos G, Kalman JM, La Meir M, Lane DA, Lebeau JP, Lettino M, Lip GYH, Pinto FJ, Thomas GN, Valgimigli M, Van Gelder IC, Van Putte BP, Watkins CL, Group ESCSD. 2020 ESC Guidelines for the diagnosis and management of atrial fibrillation developed in collaboration with the European Association of Cardio-Thoracic Surgery (EACTS). Eur Heart J. 2020.

2. Dong JZ, Sang CH, Yu RH, Long DY, Tang RB, Jiang CX, Ning M, Liu N, Liu XP, Du X, Tse HF, Ma CS. Prospective randomized comparison between a fixed '2C3L' approach vs. stepwise approach for catheter ablation of persistent atrial fibrillation. Europace. 2015;17:1798-806.

3. Verma A, Jiang CY, Betts TR, Chen J, Deisenhofer I, Mantovan R, Macle L, Morillo CA, Haverkamp W, Weerasooriya R, Albenque JP, Nardi S, Menardi E, Novak P, Sanders P, Investigators SAI. Approaches to catheter ablation for persistent atrial fibrillation. N Engl J Med. 2015;372:1812-22.

4. Takigawa M, Vlachos K, Martin CA, Bourier F, Denis A, Kitamura T, Cheniti G, Lam A, Martin R, Frontera A, Thompson N, Massoullie G, Wolf M, Escande W, Andre C, Zeng LJ, Nakatani Y, Nakashima T, Pillois X, Ramirez D, Duchateau J, Pambrun T, Sacher F, Cochet H, Hocini M, Haissaguerre M, Jais P, Derval N. Acute and mid-term outcome of ethanol infusion of vein of Marshall for the treatment of perimitral flutter. Europace. 2020;22:1252-60.

5. Valderrabano M, Peterson LE, Swarup V, Schurmann PA, Makkar A, Doshi RN, DeLurgio D, Athill CA, Ellenbogen KA, Natale A, Koneru J, Dave AS, Giorgberidze I, Afshar H, Guthrie ML, Bunge R, Morillo CA, Kleiman NS. Effect of Catheter Ablation With Vein of Marshall Ethanol Infusion vs Catheter Ablation Alone on Persistent Atrial Fibrillation: The VENUS Randomized Clinical Trial. JAMA. 2020;324:1620-8.

6. Lador A, Peterson LE, Swarup V, Schurmann PA, Makkar A, Doshi RN, DeLurgio D, Athill CA, Ellenbogen KA, Natale A, Koneru J, Dave AS, Giorgberidze I, Afshar H, Guthrie ML, Bunge R, Morillo CA, Kleiman NS, Valderrabano M. Determinants of outcome impact of Vein of Marshall Ethanol infusion when added to catheter ablation of persistent atrial fibrillation: A secondary analysis of the VENUS randomized clinical trial. Heart Rhythm. 2021.

7. Hocini M, Jais P, Sanders P, Takahashi Y, Rotter M, Rostock T, Hsu LF, Sacher F, Reuter S, Clementy J, Haissaguerre M. Techniques, evaluation, and consequences of linear block at the left atrial roof in paroxysmal atrial fibrillation: a prospective randomized study. Circulation. 2005;112:3688-96.

8. Jais P, Hocini M, Hsu LF, Sanders P, Scavee C, Weerasooriya R, Macle L, Raybaud F, Garrigue S, Shah DC, Le Metayer P, Clementy J, Haissaguerre M. Technique and results of linear ablation at the mitral isthmus. Circulation. 2004;110:2996-3002.

9. Kottkamp H, Hindricks G, Autschbach R, Krauss B, Strasser B, Schirdewahn P, Fabricius A, Schuler G, Mohr FW. Specific linear left atrial lesions in atrial fibrillation: intraoperative radiofrequency ablation using minimally invasive surgical techniques. J Am Coll Cardiol. 2002;40:475-80.

10. Spector P. Principles of cardiac electric propagation and their implications for re-entrant arrhythmias. Circ Arrhythm Electrophysiol. 2013;6:655-61. 
11. Wolf M, El Haddad M, Fedida J, Taghji P, Van Beeumen K, Strisciuglio T, De Pooter J, Lepiece C, Vandekerckhove Y, Tavernier R, Duytschaever M, Knecht S. Evaluation of left atrial linear ablation using contiguous and optimized radiofrequency lesions: the ALINE study. Europace. 2018;20:f401-f9.

12. Sawhney N, Anand K, Robertson CE, Wurdeman T, Anousheh R, Feld GK. Recovery of mitral isthmus conduction leads to the development of macro-reentrant tachycardia after left atrial linear ablation for atrial fibrillation. Circ Arrhythm Electrophysiol. 2011;4:832-7.

13. Lu X, Peng S, Xu J, Wang R, Pang L, Zhou G, Wei Y, Cai L, Wu X, Guo S, Huang D, Li W, Huang S, Liu S, Chen S. Acute conduction recurrence of mitral isthmus: Incidence, clinical characteristics, and implications. Pacing Clin Electrophysiol. 2020;43:1564-71.

14. Vlachos K, Denis A, Takigawa M, Kitamura T, Martin CA, Frontera A, Martin R, Bazoukis G, Bourier F, Cheniti G, Duchateau J, Thompson N, Massoullie G, Lam A, Wolf M, Escande W, Klotz N, Pambrun T, Sacher F, Hocini M, Haissaguerre M, Jais P, Derval N. The role of Marshall bundle epicardial connections in atrial tachycardias after atrial fibrillation ablation. Heart Rhythm. 2019;16:1341-7.

15. Valderrabano M, Chen HR, Sidhu J, Rao L, Ling Y, Khoury DS. Retrograde Ethanol Infusion in the Vein of Marshall. Circulation: Arrhythmia and Electrophysiology. 2009;2:50-6.

16. Nakashima T, Pambrun T, Vlachos K, Goujeau C, Andre C, Krisai P, Ramirez FD, Kamakura T, Takagi T, Nakatani Y, Kitamura T, Takigawa M, Roux JR, Cheniti G, Tixier R, Chauvel R, Welte N, Duchateau J, Sacher F, Cochet H, Hocini M, Haissaguerre M, Jais P, Derval N. Impact of Vein of Marshall Ethanol Infusion on Mitral Isthmus Block: Efficacy and Durability. Circ Arrhythm Electrophysiol. 2020.

17. Derval N, Duchateau J, Denis A, Ramirez FD, Mahida S, Andre C, Krisai P, Nakatani Y, Kitamura T, Takigawa M, Chauvel R, Tixier R, Pillois X, Sacher F, Hocini M, Haissaguerre M, Jais P, Pambrun T. Marshall bundle elimination, Pulmonary vein isolation, and Line completion for ANatomical ablation of persistent atrial fibrillation (Marshall-PLAN): Prospective, single-center study. Heart Rhythm. 2020.

18. Hwang C, Wu TJ, Doshi RN, Peter CT, Chen PS. Vein of marshall cannulation for the analysis of electrical activity in patients with focal atrial fibrillation. Circulation. 2000;101:1503-5.

19. Kato K, Tanaka A, Morimoto SI, Hasegawa S, Ishiguro N, Kametani R, Hattori H, Shibata N. Potential complications in patients undergoing an ethanol injection into the vein of Marshall. J Cardiovasc Electrophysiol. 2019;30:2743-50.

20. Valderrabano M, Liu X, Sasaridis C, Sidhu J, Little S, Khoury DS. Ethanol infusion in the vein of Marshall: Adjunctive effects during ablation of atrial fibrillation. Heart Rhythm. 2009;6:1552-8.

\section{Figure legends}

Figure 1. Flow diagram of patients enrollment and procedure.

Figure 2. Procedure details of 'upgraded 2C3L' approach. A. LA voltage mapping before EI-VOM during AF; B. The upper panel shows the course of VOM (white arrow), and the lower panel shows contrast staining after ethanol infusion (surrounded by white dashed line); C. LA voltage mapping shows the low-voltage area in the anterior and inferior left PVA and in the upper MI; D. Shows the RF lesion sets. RF application is not performed in the anterior and inferior left PVA and in the upper MI (white dashed line). E. LA voltage after the completion of both EI-VOM and RF ablation under sinus rhythm. LL: left lateral view; PA: posterior-anterior view; RAO: right anterior oblique view; LA: left atrium; EI-VOM: ethanol infusion into the vein of Marshall; AF: atrial fibrillation; RF: radiofrequency; SR: sinus rhythm; PVA: pulmonary vein antrum; MI: mitral isthmus.

Figure 3. Procedure details of ' $2 \mathrm{C} 3 \mathrm{~L}$ ' approach using RF energy alone. A. LA voltage mapping before ablation during AF; B. Lesion sets of initial ablation; C. LA voltage after initial '2C3L' ablation during $\mathrm{AF}, \mathrm{MI}$ block was not achieved after cardioversion; D. Additional ablation was further performed in the MI, left lateral ridge and CS to achieve MI bidirectional block. E. LA voltage under sinus rhythm after PVAI 
and bidirectional block across three ablation lines. LL: left lateral view; PA: posterior-anterior view; LA: left atrium; AF: atrial fibrillation; RF: radiofrequency; SR: sinus rhythm; CS: coronary sinus; MI: mitral isthmus.

Figure 4. K-M curve showing survival free from AF/AT recurrence with or without AAD in both groups

Table 1. Baseline characteristics of the study population.

\begin{tabular}{llll}
\hline & Group 1 $(\mathbf{n}=\mathbf{6 6})$ & Group 2 $(\mathbf{n}=\mathbf{1 2 5})$ & $\mathbf{P}$ value \\
\hline Age & $61.0 \pm 10.9$ & $61.1 \pm 10.3$ & 0.954 \\
Male gender & $47(71.2 \%)$ & $84(67.2 \%)$ & 0.570 \\
LSPAF & $32(48.5 \%)$ & $46(36.8 \%)$ & 0.118 \\
LA $(\mathrm{mm})$ & $43.6 \pm 5.5$ & $42.7 \pm 4.7$ & 0.226 \\
LV $(\mathrm{mm})$ & $50.2 \pm 6.41$ & $49.1 \pm 5.02$ & 0.208 \\
EF & $58.7 \pm 8.7$ & $59.1 \pm 7.7$ & 0.730 \\
Hypertension & $32(48.5 \%)$ & $28(22.4 \%)$ & $<0.001$ \\
CAD & $12(18.2 \%)$ & $20(16.0 \%)$ & 0.701 \\
Heart failure & $19(29.2 \%)$ & $28(22.4 \%)$ & 0.301 \\
Diabetes & $11(16.7 \%)$ & $28(22.4 \%)$ & 0.350 \\
History of stroke & $6(9.1 \%)$ & $17(13.6 \%)$ & 0.363 \\
\hline
\end{tabular}

Table 2. Procedural data and electrophysiological findings

\begin{tabular}{llll}
\hline & Group 1(n=66) & Group 2(n=125) & P value \\
\hline Total procedure time (min) & $162.4 \pm 39.7$ & $171.5 \pm 44.8$ & 0.170 \\
Radiofrequency time (min) & $11.8 \pm 8.3$ & $4.3 \pm 3.7$ & $<0.001$ \\
LPV ablation time (min) & $8.2 \pm 4.0$ & $12.6 \pm 2.9$ & $<0.001$ \\
RPV ablation time (min) & $11.7 \pm 4.4$ & $12.8 \pm 3.4$ & 0.224 \\
MAI ablation time (min) & $7.0 \pm 3.5$ & $11.8 \pm 3.5$ & $<0.001$ \\
CS ablation time (min) & $1.7 \pm 1.4$ & $1.7 \pm 1.4$ & 0.987 \\
Roofline ablation time (min) & $3.7 \pm 1.4$ & $3.6 \pm 1.1$ & 0.610 \\
CTI ablation time (min) & $5.1 \pm 2.1$ & $5.4 \pm 2.3$ & 0.523 \\
Success EI-VOM & $53(80.3 \%)$ & - & - \\
Ethanol consumption & $6.9 \pm 1.9$ & - & - \\
PVAI & $66(100 \%)$ & $125(100 \%)$ & 1.000 \\
Linear block & & & \\
MAI & $63(95.5 \%)$ & $101(80.8 \%)$ & 0.006 \\
CTI & $66(100 \%)$ & $124(99.2 \%)$ & 1.000 \\
Roofline & $66(100 \%)$ & $125(100 \%)$ & 1.000 \\
Ablation in the CS & $49(74.2 \%)$ & $95(76.0 \%)$ & 0.835 \\
SVC isolation & $3(4.5 \%)$ & $8(6.4 \%)$ & 0.601 \\
Cardioversion & $50(78.5 \%)$ & $113(90.4 \%)$ & 0.007 \\
\hline
\end{tabular}



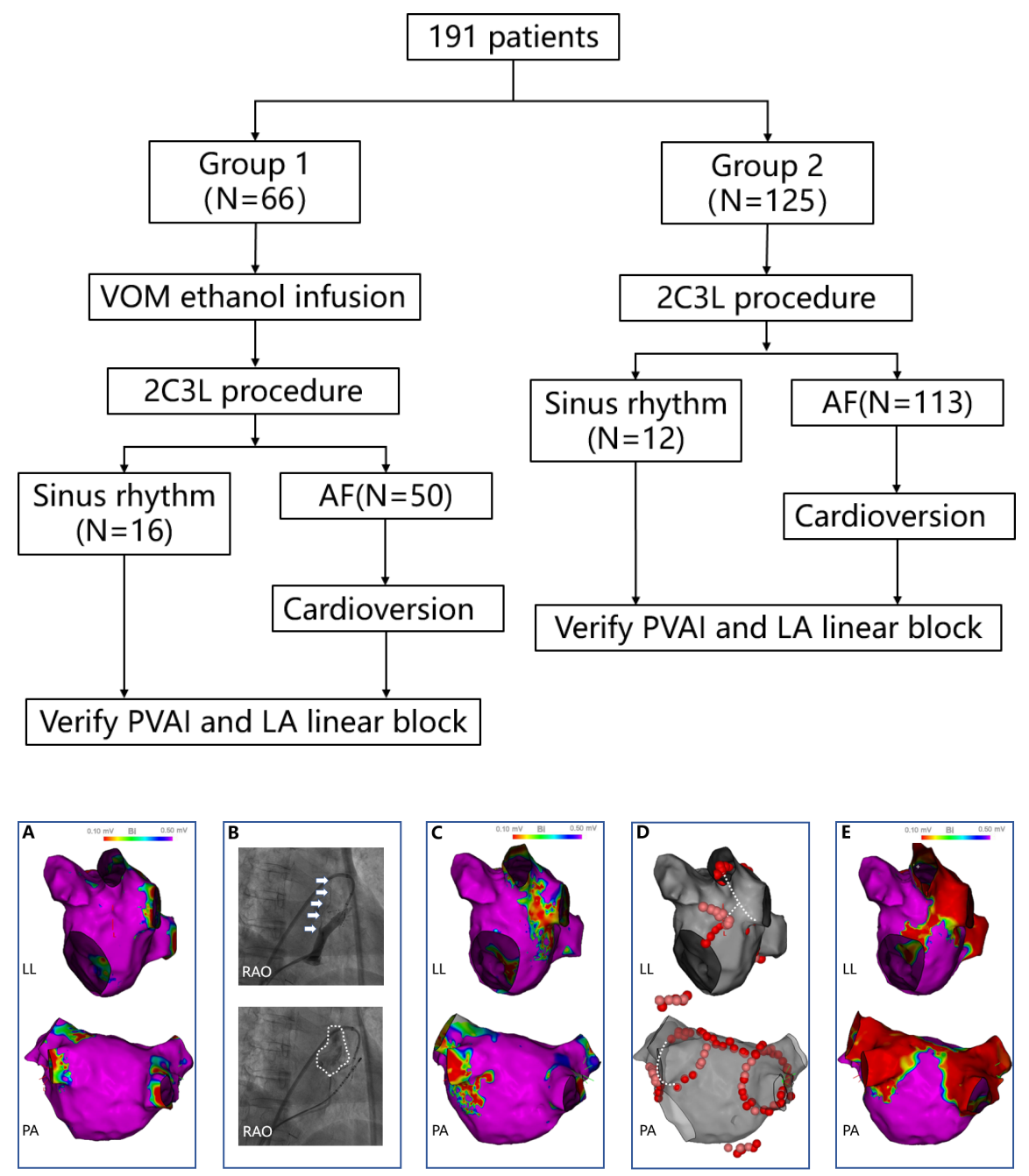

LA voltage before EI-VOM during AF
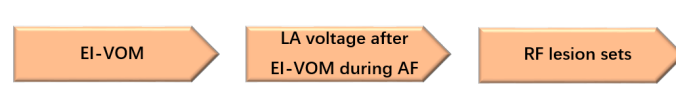

\begin{tabular}{|c|}
\hline LA voltage after \\
$\mathrm{RF}$ ablation under $\mathrm{SR}$ \\
\hline
\end{tabular}
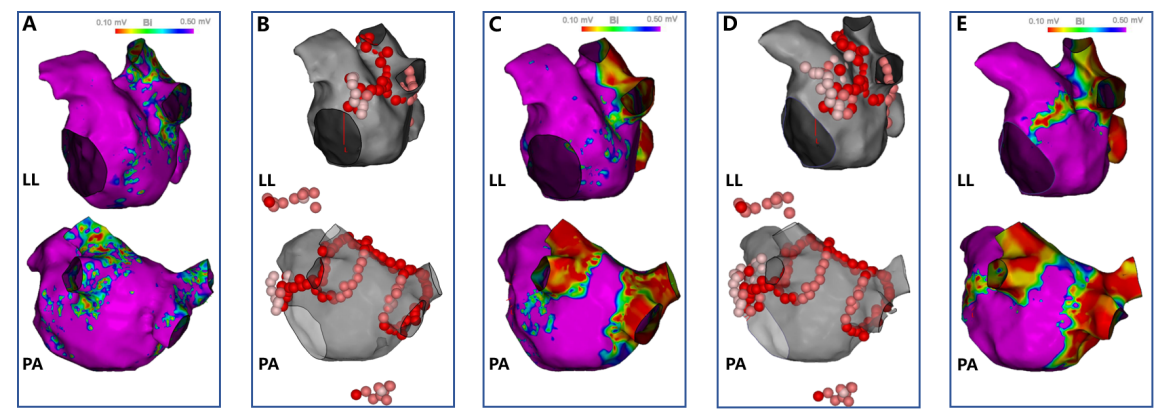

LA voltage before ablation during AF

LA voltage after initial ablation during $\mathrm{AF}$

Additional RF ablation

LA voltage after ablation under SR 


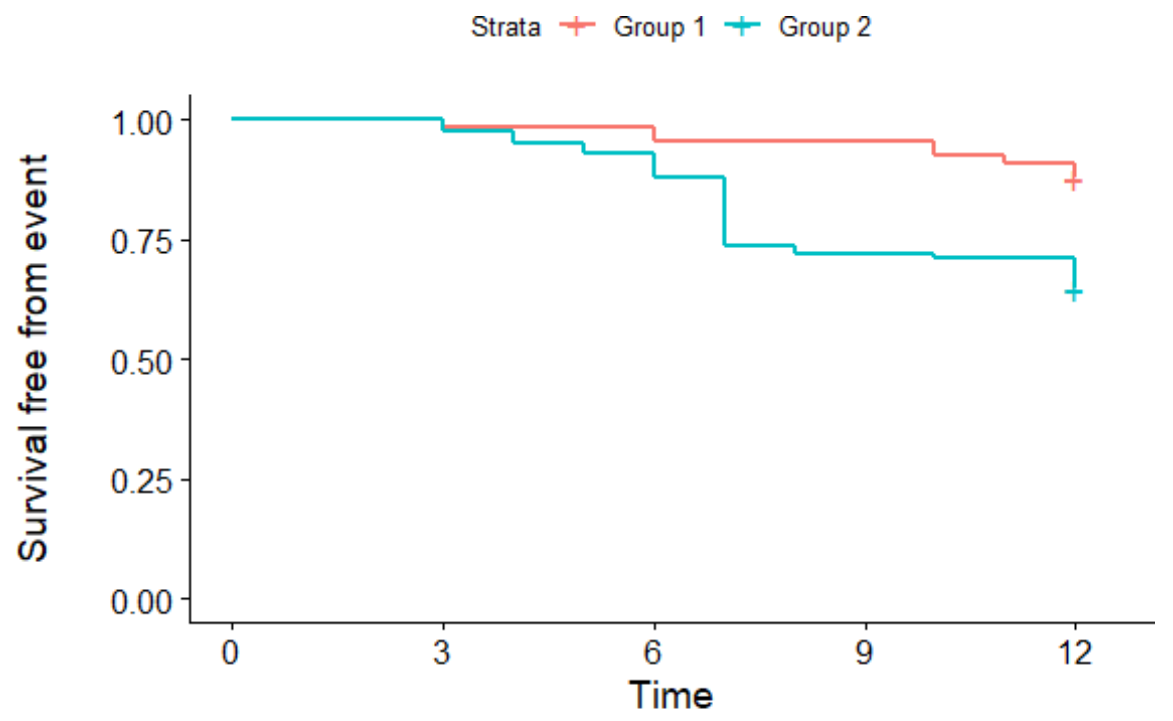

\begin{tabular}{|c|c|c|c|c|c|}
\hline \multicolumn{6}{|c|}{ Number at risk } \\
\hline $\begin{array}{l}\text { 营 Group } 1 \\
\text { 离 Group } 2\end{array}$ & $\begin{array}{r}66 \\
125 \\
\end{array}$ & $\begin{array}{c}66 \\
125 \\
\end{array}$ & $\begin{array}{c}65 \\
116\end{array}$ & $\begin{array}{l}63 \\
90 \\
\end{array}$ & $\begin{array}{l}60 \\
89\end{array}$ \\
\hline & 0 & 3 & $\begin{array}{c}6 \\
\text { Tim }\end{array}$ & 9 & 12 \\
\hline
\end{tabular}

